\title{
Hedging Barrier Options through a Log-Normal Local Stochastic Volatility Model
}

\author{
Wei Ning $^{\text {a }}$, G. Lee ${ }^{\mathrm{a}}$, N. Langrene ${ }^{\mathrm{a}}$ \\ ${ }^{a}$ CSIRO DATA61-Real Options and Financial Risk, Clayton, Victoria, 3168 \\ Email: Geoffrey.Lee@data61.csiro.au
}

\begin{abstract}
In the equity and foreign exchange (FX) markets, there has been a shift towards using nonaffine pricing models as these have been shown to produce more realistic volatility distributions and more accurately capture market dynamics. One such non-affine model is the Inverse Gamma model, which we have incorporated into a Local-Stochastic Volatility (LSV) model termed the Log-normal-LSV (LN-LSV) that can, once calibrated, accurately reproduce market prices of exotic options Langrene and Zhu [2016]. The LN-LSV model is a non-parametric combination of local volatility and stochastic volatility models, in which both the spot price and stochastic volatility follow log-normal processes. The LN-LSV model is calibrated using both the market-traded implied volatility surface and market exotic option prices.

However, while the accurate pricing of exotic options is necessary for good pricing model performance, it is also necessary for models to perform in risk management applications, where hedges are entered into to minimise risk. Therefore, the accurate calculation of the derivatives of the option price with respect to the asset or volatility (the Greeks) is also necessary for good model performance.

This paper aims to characterise the hedging performance of the Log-normal Local-Stochastic Volatility model for a variety of hedging instruments using an historical dataset consisting of daily spots and volatility surfaces for the EUR/USD market over a five-year time period. We use delta-gamma hedging for different barrier options under the LN-LSV model and compare the hedging performance with that of the Black-Scholes (BS) model. Then we use the numerical results to demonstrate that the LN-LSV model is more effective than the BS model.

We use five types of reverse knock-out options as test cases over a time period of five years. On each trading day from 2007 to 2011, the five options are firstly priced using the LN-LSV model. After pricing, each option is hedged daily until the expiry date of the option using a delta-gamma neutral scheme under both the LN-LSV and BS models. To measure the hedging performance, each profit-loss outcome forms one point of the P\&L distribution. During Jan 2007 to Dec 2011, the profit and loss of a total of 1100 traded options for each option type forms the P\&L distribution. Compared to the Black-Scholes model, the P\&L distribution of the numerical results from LN-LSV model is more symmetric and is less likely to have extreme profit-loss outliers. Thus it produces more superior hedging performance.
\end{abstract}

Keywords: Barrier option, Delta-Gamma hedging, Black-Scholes model, Log-Normal Local Stochastic Volatility model, hedging performance 


\section{INTRODUCTION}

The dynamic hedging of financial options can be improved when taking higher order Greeks into account. The most widely used first order Greek in hedging is Delta, the first order derivative of the option price with respect to the spot price. Previous work concerned with the delta hedging performance has been carried out by Boyle and Emanuel [1980]. They rebalance the portfolio at discrete times using the Black-Scholes model to ensure the hedged portfolio remains delta-neutral (i.e., the delta of the portfolio is zero at each rebalancing step). Hull and White [1987] analyse the key factors affecting the performance of delta hedging in a stochastic volatility environment. Researchers also use higher order Greeks to quantify different aspects of risk in option portfolios and attempt to make the portfolio immune to big changes in the underlying asset price. Kurpiel and Roncalli [1998] implemented both a pure delta hedge and a delta-gamma hedge the under the framework of Black-Scholes. Under a delta-gamma hedging scheme, both the delta and gamma of the portfolio remain neutral at each rebalancing step. The authors show that the delta-gamma hedging strategy improves the performance of a discrete rebalanced delta hedging and reduces the standard deviations of the net hedge costs. Then they show that in the case of delta-gamma-vega hedging scheme, where vega is also kept neutral, the stochastic volatility model outperforms the strategy based on the BS methods under some conditions.

A further improvement to the class of stochastic volatility models, where the volatility follows an independent stochastic process Gatheral [2011], is the class of Local-Stochastic Volatility (LSV) models. The class of LSV models combines the advantages of both the local volatility model (reproducing the market implied vol surface) and stochastic volatility models (reproducing market dynamics). The LN-LSV model used in this paper uses a log-normal process for the stochastic volatility component Zhu et al. [2015]. Since the LN-LSV model is more accurate in capturing the underlying dynamics of foreign-exchange spot in practise, it has been widely used by tier-one global banks for exotic option valuation in the foreign-exchange options market.

To assess the replication ability of LN-LSV model, a delta hedging backtest has been implemented Denes [2016]. The authors followed the path in Ling and Shevchenko [2016] which compares delta hedging performance under Local Volatlity and BS model. Their results show that the delta hedging performance of both the LN-LSV model and BS model are very similar. However, a delta neutral portfolio can still have non-zero gamma which is the second order derivative of option price with respect to spot price. When the spot price fluctuates widely, the unhedged movements of higher order Greeks can cause significant profit or loss. In order to compare the different hedging performances between different models, we compute the reverse knock-out barrier option prices and their first and second-order Greeks using LN-LSV and BS model respectively. We also discuss the practical implementation and the choice of hedging instruments.

In this paper, we first introduce the LN-LSV model in Section 2 We then propose the delta-gamma hedging scheme for the following five types reverse knock-out options using LN-LSV: $10 \Delta$ CallBarrier, $25 \Delta$ CallBarrier, ATMCallBarrier, $25 \Delta$ PutBarrier and $10 \Delta$ PutBarrier in Section 3 . In section 4 we use both the LN-LSV and the BS model to price these options and compute their Greeks respectively on each trading day through the life cycle. We then analyse and compare the hedging performance of these two models.

\section{Log-Normal Local-Stochastic Volatility Model}

The LN-LSV model is a non-parametric combination of local volatility and stochastic volatility models. In this model, we assume that both spot price $S_{t}$ and volatility $\sigma_{t}$ follow their log-normal stochastic processes Zhu et al. [2015].

$$
\begin{array}{ll}
d S_{t}=\left[r_{d}(t)-r_{f}(t)\right] S_{t} d t+L\left(S_{t}, t\right) \sigma_{t} S_{t} d W_{t}^{1}, & S_{0}=s, \\
d \sigma_{t}=\kappa\left(\theta-\sigma_{t}\right) d t+\lambda \sigma_{t} d W_{t}^{2}, & \sigma_{0}=v, \\
\mathbb{E}\left[d W_{t}^{1} \cdot d W_{t}^{2}\right]=\rho d t . &
\end{array}
$$

where $r_{d}(t)$ is domestic interest rate and $r_{f}(t)$ is foreign interest rate, both of which are assumed to be of term structures. We assume that the other stochastic parameters $\kappa, \theta, \lambda$ and $\rho$ in LN-LSV model also have term structures. $L\left(S_{t}, t\right)$, termed the leverage function, representing the ratio between local volatility and the expectation of stochastic volatility conditional on the current asset price $S_{t}$. We calibrate the leverage function $L$ numerically using market data. 
Table 1. Reverse knock-out options

\begin{tabular}{lcl}
\hline \hline $10 \Delta$ Call Barrier & A reverse Up-And-Out Call equivalent to a 10 $\Delta$ Call & Barrier $=1.025 K$ \\
$25 \Delta$ Call Barrier & A reverse Up-And-Out Call equivalent to a $25 \Delta$ Call & Barrier $=1.025 K$ \\
ATM Call Barrier & A reverse Up-And-Out Call equivalent to a ATM Call & Barrier $=1.025 K$ \\
$10 \Delta$ Put Barrier & A reverse Down-And-Out Put equivalent to a $10 \Delta$ Put & Barrier $=0.975 K$ \\
$25 \Delta$ Put Barrier & A reverse Down-And-Out Put equivalent to a $25 \Delta$ Put & Barrier $=0.975 K$ \\
\hline
\end{tabular}

\section{Dynamic Delta-Gamma Hedging}

In constructing the Delta-Gamma hedging scheme, we make the assumptions that the market is arbitrage free, perfectly liquid and frictionless, and we can take any fractional short or long position in any asset. In order to keep the portfolio Delta-Gamma neutral, we need to rebalance the hedging instruments on each trading day.

\subsection{Delta-Gamma Neutral Hedging}

Each barrier option is Delta-Gamma hedged daily using both the LN-LSV model and the BS model until the expiry. For each barrier option, we construct a delta-gamma neutral portfolio which contains a short position $N_{O}$ of the barrier option, a long position $N_{h}$ of hedging instrument and $N_{S}$ underlying asset. When rebalancing, a Delta-Gamma neutral portfolio should satisfy the following constraints:

$$
\begin{gathered}
\Delta_{\text {Portfolio }}=\Delta_{S} N_{S}-\Delta_{O} N_{O}+\Delta_{h} N_{h}=0, \\
\Gamma_{\text {Portfolio }}=\Gamma_{S} N_{S}-\Gamma_{O} N_{O}+\Gamma_{h} N_{h}=0 .
\end{gathered}
$$

where $N_{h}$ and $N_{S}$ are the net positions of hedging instrument and stock in this portfolio, $\Delta_{O}$ and $\Gamma_{O}$ denote delta and gamma of the barrier option, $\Delta_{h}$ and $\Gamma_{h}$ denote delta and gamma of the hedging instrument respectively. For the gamma and delta of the stocks, we have $\Gamma_{S}=0$ and $\Delta_{S}=1$. Assuming the short position of barrier option $N_{O}=1$, we can solve for $N_{h}$ and $N_{S}$ from the linear system (23). Therefore we have:

$$
N_{h}=\frac{\Gamma_{O}}{\Gamma_{h}}, \quad N_{S}=\Delta_{O}-\Delta_{h} \frac{\Gamma_{O}}{\Gamma_{h}} .
$$

\subsection{Hedging Instrument}

In this paper, we focus on hedging the five reverse knock-out barrier options listed in Table (1). The maturities of these options are 3 months.

To select the hedging instrument, Molchan and Rouah hedged a one-month Up-and-Out call option with a vanilla call of identical features Molchan and Rouah [2011]. Raju [2012] applied Delta-Gamma hedgeing for vanilla call options by another vanilla call option with a different strike using BS model. In 2009, Engelmann et al. proposed Delta-Vega hedging on Down-and-Out barrier options where they adopted ATM vanilla option as the hedging instrument Engelmann et al. [2009]. Besides dynamic hedging, Derman et al. introduced static hedging through a portfolio of vanilla options with various strikes and maturities Derman et al. [1995].

From the these papers, we can see that vanilla option is a reasonable candidate as our hedging instrument. First of all, vanilla options are similar to barrier options except when the barrier is triggered. Secondly, a combination of vanilla options with different strikes can generate any desirable piecewise linear payoffs. Furthermore, the Delta and Gamma of vanillas are straightforward to compute and well-behaved Derman and Kani [1997]. The hedging instruments used in this paper are listed in Table 2 We select vanilla options as the hedging instrument with the same maturities and strikes as the barrier options.

\subsection{Delta-gamma Hedging}

We rebalance each portfolio daily until expiry. Since the interest is accumulated continuously, when Monday is the trading day, 3-day ( Friday and weekend) interest should be accrued in the portfolios, otherwise 1-day interest is applied Ling and Shevchenko [2016]. Let $\{t\}_{i}$ denote the trading days where $i \in\{1, \ldots, N\}$, and $N$ is the total number of trading days from time 0 to $T$. $C$ is the price of barrier option hedged at time $t_{0}, S^{i}$ is the price of underlying stock at time $t_{i}, H^{i}$ is the price of hedging instrument (vanilla option) at time $t_{i}$. $N_{h}^{i}$ and $N_{S}^{i}$ are net positions of hedging instrument and stock in the portfolio at time $t_{i}$, defined as Equation (4). We test the hedging performance with the following steps: 
Table 2. Hedging instruments in LN-LSV and BS model

\begin{tabular}{ll}
\hline \hline \multicolumn{1}{c}{ Target Barrier Options } & Hedging Instrument \\
\hline $10 \Delta$ Call Barrier & Vanilla $10 \Delta$ Call \\
$25 \Delta$ Call Barrier & Vanilla $25 \Delta$ Call \\
ATM Call Barrier & Vanilla ATM Call \\
$10 \Delta$ Put Barrier & Vanilla $10 \Delta$ Put \\
$25 \Delta$ Put Barrier & Vanilla $25 \Delta$ Put \\
\hline
\end{tabular}

1. At $t_{0}$, we short 1 unit barrier option and long $N_{s}^{0}$ stock and $N_{h}^{0}$ hedging instrument. The cash position at initial time $t_{0}$ is: $P_{0}=C-N_{s}^{0} S^{0}-N_{h}^{0} H^{0}$.

2. At the next trading day $t_{1}$, we start the first rebalancing by longing $N_{S}^{1}$ shares and $N_{h}^{1}$ hedging instrument, thus this results in the change $\left(N_{S}^{0}-N_{S}^{1}\right) S^{1}+\left(N_{h}^{0}-N_{h}^{1}\right) H^{1}$. At the same time, the interest accrued / charged on cash position $P_{0}$ equals to $\left(e^{a r_{d}\left(t_{0}\right)}-1\right) P_{0}$ and dividend yield received / paid on underlying stock is $\left(e^{a r_{f}\left(t_{0}\right)}-1\right) N_{S}^{0} S^{0}$, where $a$ is the time (in years) between $t_{0}$ to $t_{1}$. If trading day $t_{1}$ is a Monday, then $a=3 / 365$, otherwise, $a=1 / 365$. To sum up the changes in the portfolio, we get the cash position at $t_{1}: P_{1}=P_{0}+\left(e^{a r_{d}\left(t_{0}\right)}-1\right) P_{0}+\left(e^{a r_{f}\left(t_{0}\right)}-1\right) N_{S}^{0} S^{0}+\left(N_{S}^{0}-N_{S}^{1}\right) S^{1}+\left(N_{h}^{0}-N_{h}^{1}\right) H^{1}$.

3. On the $i$-th trading day $t_{i}$, where $i \in\{1, \ldots, N-1\}$, the cash position is given by $P_{i}=e^{a r_{d}\left(t_{i-1}\right)} P_{i-1}+\left(e^{a r_{f}\left(t_{i-1}\right)}-1\right) N_{S}^{i-1} S^{i-1}+\left(N_{S}^{i-1}-N_{S}^{i}\right) S^{i}+\left(N_{h}^{i-1}-N_{h}^{i}\right) H^{i}$.

4. At maturity $t_{N}=T$, we sell both underlying shares and hedging instrument. The final cash position $P_{N}=e^{a r_{d}\left(t_{N-1}\right)} P_{N-1}+\left(e^{a r_{f}\left(t_{N-1}\right)}-1\right) N_{S}^{N-1} S^{N-1}+N_{S}^{N-1} S^{N}+N_{h}^{N-1} H^{N}$. One the other side, the payoff of the barrier option is $X=\max \left(0, S^{N}-K\right)$ for call options and $X=\max \left(K-S^{N}, 0\right)$ for put options if it is not knocked out, otherwise $X=0$.

5. At time expiry $T$, the hedging error is $P_{N}-X$ because of the short position of barrier option.

\section{Numerical Test}

In this section, we backtest the performance of delta-gamma hedging using historic daily EURUSD data from 2007/01/01 to 2011/12/30. These market data include: the spot price, implied volatilities for at the money (ATM) options, 10 and 25 delta risk reversals, 10 and 25 delta butterflies and zero coupon rates for the domestic (USD) and foreign (EUR) currencies. From these data, we can calculate strikes of the options from BS formula, and interpolate market implied volatilities using natural cubic spline Press [2007] for each trading day.

Figures 1 to 5 depict the profit and loss $(\mathrm{P} \& \mathrm{~L})$ distributions of Delta-Gamma hedging under frameworks of BS and LN-LSV models. The sample means, standard deviations and other statistics for these 5 barrier options are summarised in Tables (3- 7 ).

Table 3. 10 $\Delta$ CallBarrier Summaries

\begin{tabular}{lccccccc}
\hline \multicolumn{1}{c}{ Model } & Mean. & Median. & Max & Min & Standard Dev. & Skewness & Kurtosis \\
\hline \hline BS & -0.000218 & $1.72 \mathrm{E}-07$ & 0.01941 & -0.037906 & 0.003015 & -5.726914 & 60.25944 \\
LN-LSV & -0.000246 & $2.08 \mathrm{E}-05$ & 0.02034 & -0.041326 & 0.003377 & -4.795989 & 48.61074 \\
\hline
\end{tabular}

Table 4. 25 $\Delta$ CallBarrier Summaries

\begin{tabular}{lccccccc}
\hline \multicolumn{1}{c}{ Model } & Mean. & Median. & Max & Min & Standard Dev. & Skewness & Kurtosis \\
\hline \hline BS & -0.00761 & -0.000105 & 0.293873 & -4.998658 & 0.166492 & -26.85515 & 767.2382 \\
LN-LSV & -0.001707 & -0.000205 & 0.016951 & -0.155152 & 0.007682 & -10.94762 & 185.6279 \\
\hline
\end{tabular}




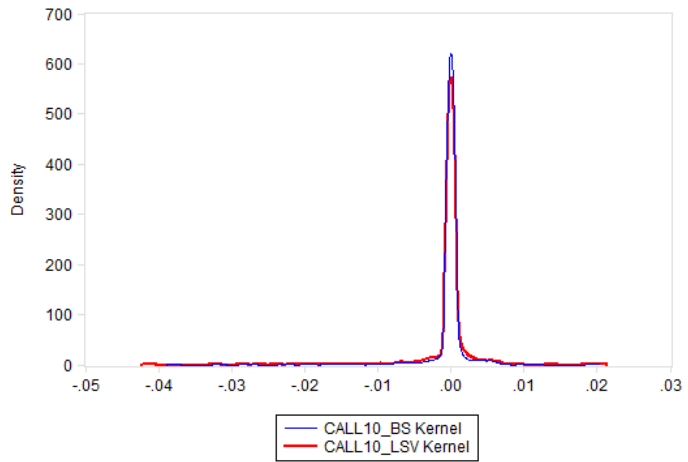

Figure 1. P\&L distribution for $10 \Delta$ CallBarrier

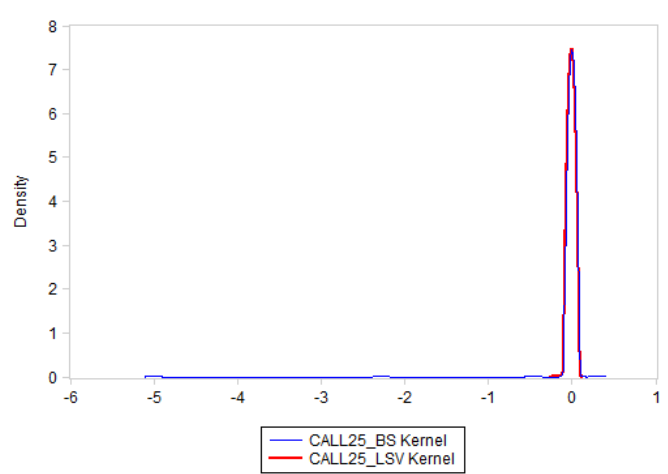

Figure 2. $\mathrm{P} \& \mathrm{~L}$ distribution for $25 \Delta$ CallBarrier

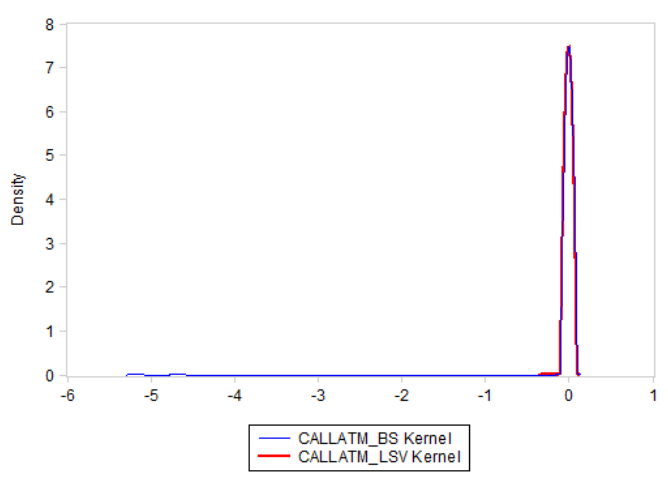

Figure 3. P\&L distribution for ATMCallBarrier

Table 5. ATMCallBarrier Summaries

\begin{tabular}{lccccccc}
\hline \multicolumn{1}{c}{ Model } & Mean. & Median. & Max & Min & Standard Dev. & Skewness & Kurtosis \\
\hline \hline BS & -0.009302 & -0.00017 & 0.038319 & -5.179844 & 0.210417 & -23.47105 & 553.3903 \\
LN-LSV & -0.000516 & $-5.48 E-05$ & 0.017902 & -0.242595 & 0.010443 & -21.66694 & 494.7996 \\
\hline
\end{tabular}

Table 6. 25 $\Delta$ PutBarrier Summaries

\begin{tabular}{lccccccc}
\hline Model & Mean. & Median. & Max & Min & Standard Dev. & Skewness & Kurtosis \\
\hline \hline BS & -0.000809 & $-4.86 E-05$ & 0.004682 & -0.043284 & 0.004064 & -6.408469 & 48.63383 \\
LN-LSV & -0.000936 & -0.000108 & 0.004846 & -0.041772 & 0.004286 & -5.988037 & 42.52505 \\
\hline
\end{tabular}

Table 7. $10 \Delta$ PutBarrier Summaries

\begin{tabular}{lccccccc}
\hline \multicolumn{1}{c}{ Model } & Mean. & Median. & Max & Min & Standard Dev. & Skewness & Kurtosis \\
\hline \hline BS & -0.000103 & $8.25 E-06$ & 0.013338 & -0.036021 & 0.002469 & -8.311014 & 105.2769 \\
LN-LSV & -0.00024 & $-1.88 E-05$ & 0.009129 & -0.041599 & 0.002633 & -9.492582 & 116.838 \\
\hline
\end{tabular}




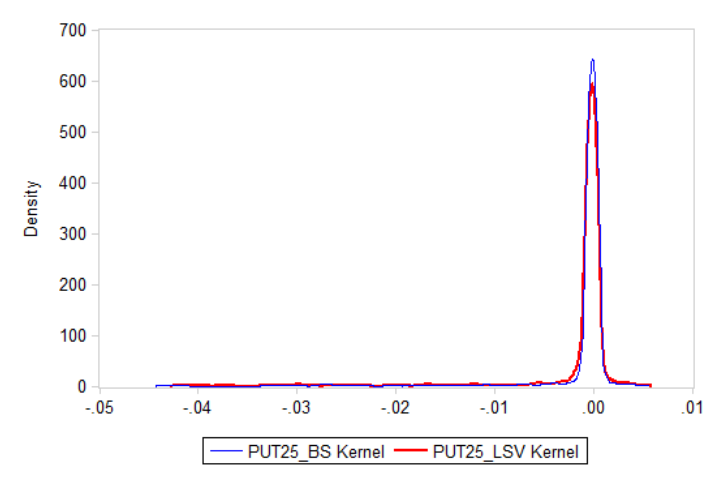

Figure 4. P\&L distribution for $25 \Delta$ PutBarrier

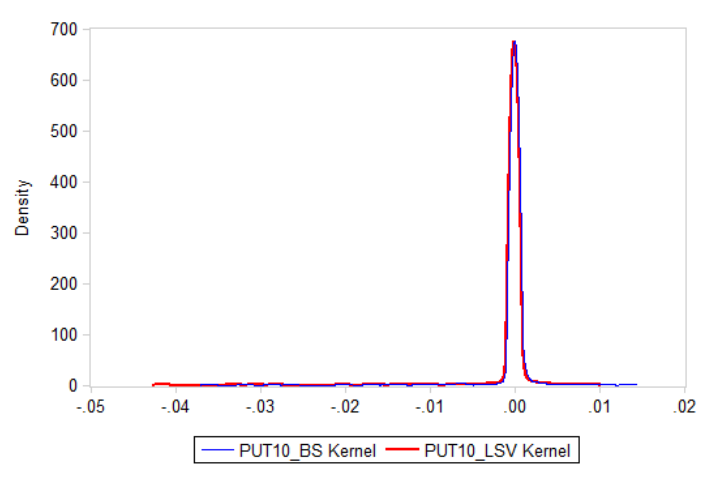

Figure 5. P\&L distribution for $10 \Delta$ PutBarrier

\section{CONCLUSIONS AND RECOMMENDATIONS}

The result from this experiment shows that, compared to the BS model, the hedging performance of the LN-LSV model is more symmetric, stable and less likely to have outliers, which can be seen in Figure 2 and Figure 3. In these two figures, the peaks in the denstiy plots of the LN-LSV and the BS models are very similar, however the BS model plots tend to have long tails.

In terms of the statistics of hedging errors, there is no significant difference between the means in LN-LSV and BS model. However, for 4 out of 5 options, the medians of hedging errors of BS model are closer to zero and less than those of LN-LSV model. Meanwhile, for 25 $\Delta$ CallBarrier and ATMCallBarrier, the standard deviations of the LN-LSV model are less than those of the BS model. While for $10 \Delta$ CallBarrier, $25 \Delta$ PutBarrier and $10 \Delta$ PutBarrier, the standard deviations of the two models are very similar.

The most significant difference between these two models is the outlier of the plots. For the options $25 \Delta$ CallBarrier and ATMCallBarrier, the hedging error in the BS model can be as large as -5 , while the minima for the LN-LSV model are only -0.155 and -0.24 , see Table 4 and Table 5 . Especially for ATMCallBarrier, the statistics of the LN-LSV model are overall better than those of the BS model. This tendency of the BS model having significant outliers is indicated in the skewness and kurtosis. Except the option 10 $\Delta$ PutBarrier, skewnesses and kurtosises of LN-LSV model are significantly smaller than those of BS model, which means that the hedging errors of LN-LSV models are more stable and symmetric.

Our numerical experiment shows that the hedging performance of the LN-LSV model is very similar to or better than that of the BS model. For some options, the error from the LN-LSV model is stable and has less outliers. Thus this Delta-Gamma hedging process demonstrates the advantage of the LN-LSV model in pricing the Greeks of Barrier options.

However, compared to Delta hedging, Delta-Gamma hedging results relatively larger hedging errors. One cause for this phenomenon is the unstable behaviour of Gamma, especially when the underlying price is near the barriers Derman and Kani [1997]. Another reason which leads to larger hedging errors is that there is no standard or unique way for choosing the hedging instruments. However the hedging performance is highly dependent on the choice of instruments. Further research can be done for selecting optimal hedging instruments to minimise the hedging errors.

\section{ACKNOWLedgments}

The authors would like to acknowledge that Michael Denes implemented the initial delta hedging computer code which the current delta-gamma hedging work is based on. Special thanks to Zili Zhu, Owens Bowie and Thomas Lo for their work in developing Log-Normal Stochastic Local Volatility model. A grateful thanks to Wen Chen for revising this paper. 


\section{REFERENCES}

Boyle, P. P. and D. Emanuel (1980). Discretely adjusted option hedges. Journal of Financial Economics 8(3), 259-282.

Denes, M. C. (2016). Hedging fx risk through a log-normal local-stochastic volatility model.

Derman, E., D. Ergener, and I. Kani (1995). Static options replication. The Journal of Derivatives 2(4), 78-95.

Derman, E. and I. Kani (1997). The ins and outs of barrier options: Part 1. Derivatives Quarterly 3, 73-80.

Engelmann, B., M. R. Fengler, and P. Schwendner (2009). Hedging under alternative stickiness assumptions: an empirical analysis for barrier options. The Journal of Risk 12(1), 53.

Gatheral, J. (2011). The volatility surface: a practitioner's guide, Volume 357. John Wiley \& Sons.

Hull, J. and A. White (1987). Hedging the risks from writing foreign currency options. Journal of International money and Finance 6(2), 131-152.

Kurpiel, A. and T. Roncalli (1998). Option hedging with stochastic volatility.

Langrene, N. Lee, G. and Z. Zhu (2016). Switching to non-affine stochastic volatility: A closed-form expansion for the inverse gamma model. International Journal of Theoretical and Applied Finance 19, 1650031.

Ling, T. G. and P. V. Shevchenko (2016). Historical backtesting of local volatility model using aud/usd vanilla options. The ANZIAM Journal 57(03), 319-338.

Molchan, J. and F. D. Rouah (2011). Where?s my delta? The Journal of Wealth Management 13(4), 68-76.

Press, W. H. (2007). Numerical recipes 3rd edition: The art of scientific computing. Cambridge university press.

Raju, S. (2012). Delta gamma hedging and the black-scholes partial differential equation (pde). Journal of Economics and Finance Education 11(2).

Zhu, Z., G. Lee, N. Langrene, B. Owens, and L. Thomas (2015). Pricing barrier options with a log-normal local- stochastic volatility model. 